\title{
Anterior Segment Scleral Fluorescein Angiography in the Evaluation of Ciliary Body Neoplasm: Two Case Reports
}

\author{
Amir H. Marvasti ${ }^{a}$ Jesse Berry ${ }^{b}$ Maria E. Sibug Saber ${ }^{b}$ \\ Jonathan W. Kim ${ }^{\text {b }}$ Alex S. Huang ${ }^{c}$ \\ ${ }^{a}$ Shiley Eye Institute, Department of Ophthalmology, University of California, \\ San Diego, Calif., ' USC Eye Institute, Keck School of Medicine, University of Southern \\ California, and 'Doheny Eye Institute, David Geffen School of Medicine, University of \\ California, Los Angeles, Calif., USA
}

\section{Key Words}

Scleral angiography · Ocular tumor · Ciliary body

\begin{abstract}
Introduction: Anterior segment tumors can be difficult to detect until tumor growth is substantial enough to cause local signs or symptoms. Earlier detection may result in improved outcomes, particularly the ability to option for globe-conserving therapy. Multiple diagnostic modalities such as ultrasound or optical coherence tomography exist to aid for earlier detection of ciliary body tumors, but they also have limitations. Here we describe the potential for scleral angiography as an adjunctive modality to assist in evaluating anterior segment ciliary body tumors. Case Presentations: A 61-year-old Caucasian male and a 57-year-old Hispanic female presented for ciliary body tumor evaluation. The Caucasian male notably had abnormal scleral, episcleral, and conjunctival vessels in the affected eye. Scleral angiography was performed in both cases with the abnormal vasculature highlighted in the Caucasian male. The Hispanic female did not demonstrate abnormal scleral angiographic patterns. Notably, the Caucasian male also had regions of abnormal scleral angiography arising in locations of otherwise normal appearing sclera. Both patients had the affected eyes enucleated. Histology of the enucleated eyes demonstrated a ciliary body melanoma in the Caucasian male associated with abnormal vascular and tumor infiltration of the scleral bed. The Hispanic female had a pigmented ciliary body adenoma without involvement of the scleral bed. Conclusion: With limited sample size, scleral angiography has the potential to detect abnormal scleral
\end{abstract}

\section{KARGER}

Alex S. Huang, MD, PhD

Department of Ophthalmology, Doheny Eye Institute

David Geffen School of Medicine, University of California

1355 San Pablo Street, Los Angeles, CA 90033 (USA)

E-Mail Ahuang@Doheny.org 
Marvasti et al.: Anterior Segment Scleral Fluorescein Angiography in the Evaluation of Ciliary Body Neoplasm: Two Case Reports

vascular patterns in otherwise normal appearing sclera in cases of ciliary body tumor with scleral vascular invasion.

(C) 2016 The Author(s)

Published by S. Karger AG, Basel

\section{Introduction}

Among the various types of anterior segment ocular neoplasms, ciliary body tumors pose a unique diagnostic challenge as they can go undetected on routine ophthalmic examination. For instance, in contrast to tumors confined to the iris, which are typically visible and detected early, ciliary body tumors often go undetected until they are large and in more advanced stages [1]. This is mainly due to the tumor being hidden from view behind the iris and/or lens. As such, ciliary body melanomas are generally considered to have a worse prognosis due to the fact that they are larger at diagnosis $[2,3]$. The presence of a dilated episcleral vessel that is potentially a 'sentinel vessel' can be a clue to the physician regarding a ciliary body lesion and aid in early diagnosis [4].

Given difficulty in detecting ciliary body tumors, multiple diagnostic tests exist to aid in their evaluation. Ultrasound biomicrosopy (UBM) has been the main imaging modality for evaluating anterior segment ocular tumors [3]. While undoubtedly providing quality imaging of the ciliary body, UBM requires a skilled operator. Anterior segment optical coherence tomography (OCT) has also been used to image iris and ciliary body tumors [5]. OCT allows for an easier and faster clinical examination without direct contact to the eye and potentially superior resolution of anterior segment anatomy and tumors. However, OCT has limited ability to penetrate behind the iris to the deeper ciliary body. Even with all of these advances, ciliary body tumors still pose a diagnostic challenge, partly because of a lack of a broadly used screening method.

Herein we describe scleral angiography as a possible screening and imaging modality for occult ciliary body tumors. Anterior segment fluorescein angiography was first introduced for the limited purposes of revealing pathological findings in iris vessels [6]. For the sclera, Watson and colleagues [7-9] introduced scleral angiography for identification and study of subclinical necrotizing scleritis. As such, anterior segment fluorescein angiography is a method that maybe useful to assess the vascular pattern of anterior segment tumors. We present two cases of confirmed ciliary body tumors, one with and one without histological evidence of scleral/vascular involvement, and describe the patterns seen on scleral angiography.

\section{Case Presentations}

\section{Patient 1}

A 61-year-old Caucasian male presented with a 6-month history of vision loss. He was initially seen in an outside emergency room and diagnosed with conjunctivitis based on a red eye. He was subsequently diagnosed with cataract and glaucoma and started on pressure-lowering drops. One week prior to referral, he was noted to have iris abnormalities and was referred for a presumed ciliary body mass in the right eye. Complete review of system was unremarkable. Past medical and ocular history was significant for hypertension, hypercholesterolemia, and glaucoma. Medications included brinzolamide twice daily in the right eye, travaprost daily in the right eye, enalapril, furosemide, and gemfibrozil. 
Marvasti et al.: Anterior Segment Scleral Fluorescein Angiography in the Evaluation of Ciliary Body Neoplasm: Two Case Reports

In the right eye, visual acuity was hand motions. The intraocular pressure was $48 \mathrm{~mm}$ Hg. The right pupil was irregular, nonreactive, and an afferent pupillary defect was noted by reverse. Slit lamp examination revealed large dilated conjunctival and episcleral vessels nasally (fig. 1a, g) intermixed with regions of grossly normal appearing sclera. The anterior chamber exam showed an amelanotic mass with intralesional dilated vasculature in the superonasal quadrant which appeared to have eroded through the iris root into the anterior chamber focally shallowing the anterior chamber (fig. 1b). There was 360 degrees of iris neovascularization (fig. 1b) and a cataract with posterior synechiae. There was no view to the right eye on dilated fundus exam. Complete examination of the left eye was unremarkable.

Further workup for the lesion included UBM and B-scan ultrasound. UBM showed a tumor that measured $18.44 \mathrm{~mm}$ in the transverse meridian, $15.91 \mathrm{~mm}$ in the longitudinal meridian, and $10.22 \mathrm{~mm}$ in height. B-scan demonstrated a dome-shaped lesion. There was low to medium internal reflectivity on A-scan.

Scleral angiography was performed. This technique is analogous to intravenous fluorescein angiography for the retina [10]. The patient was consented and the eye marked. Intravenous access was obtained through a butterfly needle in a peripheral vein. The patient was placed in front of a Heidelberg Spectralis (HRA+OCT) and imaged with a 55-degree lens. Three milliliters of $10 \%$ fluorescein (Akorn) was delivered intravenously and angiographic images were obtained. The camera focused on the external sclera. Scleral angiography clearly highlighted the abnormal vascularity in the nasal region of the right eye, which was also seen clinically. Early arterial phase ( $<20 \mathrm{~s}$ [7]) led to arteriovenous phase $(<35 \mathrm{~s}$ [7]) culminating in eventual leakage over the region (fig. 1c-e). Both eyes were imaged, with the left (uninvolved eye) serving as a normal control to the right. The left eye showed normal organized vascular arcades with clear intervening regions (fig. 1f). While large, tortuous, and abnormal vessels in the right eye were apparent clinically in the conjunctiva and sclera, scattered areas still appeared white and reminiscent of normal sclera (fig. 1g; black asterisk). However, when viewed under scleral angiography, these areas were actually abnormal with a deep network of lacy and dilated vessels suggestive of further pathology (fig. 1h; white asterisk).

\section{Patient 2}

A 57-year-old Hispanic female was evaluated for decreased vision in the right eye for 2 years. A broad review of systems was unremarkable except for an episode of syncope a month prior to her visit. A computed tomography (CT) imaging of brain was normal. She had no significant past medical or ocular history aside from pterygia in both eyes. Her medications included meclizine, and she was not taking any topical medications.

In the right eye, visual acuity was bare hand motions. The pressure was $17 \mathrm{~mm} \mathrm{Hg}$. The pupil was irregular and minimally reactive. Slit lamp examination revealed trace hyperemia of the conjunctiva and a pigmented lesion inferotemporally behind the iris, involving the iris root, and with anterior bowing of the iris (fig. 2a, b). There was neovascularization of the iris in this quadrant, and mild ectropion uvea was noted. With dilation, the inferotemporal pigmented lesion (fig. 2a), which was more readily apparent, was associated with a superonasally subluxed white cataractous lens (fig. 2a). Complete examination of the left eye was unremarkable.

Workup for the observed pigmented lesion included UBM, which showed a $7.29 \times 10.33$ $\times 6.05 \mathrm{~mm}$ round ciliary body-based mass with abnormal signal intensity in the right globe. The patient declined a biopsy for diagnosis; however, an MRI was done, which showed ab- 
Marvasti et al.: Anterior Segment Scleral Fluorescein Angiography in the Evaluation of Ciliary Body Neoplasm: Two Case Reports

normal signal intensity on both T1-weighted and flair sequences in the right eye near the lens.

Scleral angiography was performed as described above. Scleral angiography in patient 2 demonstrated early arterial phase that quickly included venous filling with pinpoint leaks that expanded gradually with time (fig. 2c-h). Both eyes were imaged on separate occasions, with the left (uninvolved eye) serving as a normal control to the right. Both eyes demonstrated similar and normal scleral angiographic images and patterns.

\section{Histopathology for Patients 1 and 2}

Given the presence of ciliary body lesions with poor visual prognosis, enucleations were performed in the affected eyes of both patients. For patient 1, histopathologic examination confirmed the diagnosis of a ciliary body melanoma showing pigment deposition intermixed with spindle cells (fig. 3a). There was no evidence of extrascleral or optic nerve invasion. The tumor bordered the scleral wall and points of tumor and vascular infiltration into the sclera were identified (fig. 3b) leading to scattered regions of abnormal larger (fig. 3c) and smaller (fig. 3d) intrascleral vessels with associated abnormal cells.

For patient 2, histopathologic examination revealed a ciliary epithelial adenoma (fig. 3e) with resultant compression of the iris, iridocorneal adhesions, and obliteration of the angle. Immunohistochemistry supported this diagnosis (data not shown). The location of the tumor was notably anterior to the ciliary body resulting in no contact with the scleral wall. The sclera demonstrated normal collagen bundles and vasculature throughout (fig. 3f).

\section{Discussion}

Ciliary body tumors are occult and often difficult to detect until they are large enough to cause signs and symptoms [1]. Ciliary body melanomas are potentially more dangerous as they often go undetected behind the iris until they have grown to a large size [2,3]. Early visualization of the tumor may allow for globe conservation as opposed to enucleation [11, 12]. In this report, we illustrate the potential of scleral angiography as an adjunctive tool for ciliary body tumor evaluation.

Scleral angiography belongs to a family of angiographic methods including intravenous angiograms for the retina/choroid [10], anterior segment angiograms for the iris vasculature $[13,14]$, and aqueous angiography (manuscript under review), which is a method to detect aqueous humor outflow pathways. In all cases, tracers, typically fluorescent, are introduced and then visualized with specific excitation/emission profiles of the tracers using white light with filters or wavelength-specific lasers for excitation. Each of these methods provides high-quality images, and scleral angiography has been previously described as having utility in evaluating subclinical scleritis [7-9]. An advantage for scleral angiography for ciliary body tumor evaluation is that the capability to perform scleral angiography may be more widely available in clinics worldwide where access to more advanced technologies such as UBM and OCT maybe limited.

In this report, evaluating two patients, one with and one without tumor and vascular infiltration of the sclera, the potential use of scleral angiography in early detection of ciliary body tumors is described. Here, patient 1 demonstrated abnormal patterns seen on scleral angiography in normal appearing sclera albeit surrounded by abnormal vessels. Patient 2 served as a control to patient 1 , offered an example of what normal scleral angiography appears like, and also provided a lesson that scleral angiography is not foolproof. If the tumor does not abut nor involve the sclera, scleral angiography may not be informative. 
Marvasti et al.: Anterior Segment Scleral Fluorescein Angiography in the Evaluation of Ciliary Body Neoplasm: Two Case Reports

Weaknesses of this case series include number of patients studied and the fact that patient 1 presented initially with obvious vascular changes. With an initial description of a method, a more overt case was started with, as a normal appearing eye requiring tumor evaluation with enucleation and pathological assessment is rare. Nevertheless, scleral angiography was able to detect vascular abnormalities in focal regions of the sclera that otherwise appeared normal at the slit lamp. The identity of these angiographic findings as secondary to intrascleral tumor extension with an abnormal scleral vascular bed was confirmed by histology after enucleation. Extending from this, we speculate that prior to the development of clinically apparent vascular abnormalities, scleral angiography might have had the ability to demonstrate angiographic alterations for the purposes of early detection.

Furthermore, for any tracer study, the final result is only as good as the advantageous and disadvantageous characteristics of each tracer (size, charge, $\mathrm{pH}$, etc.). Recall that introduction of indocyanine green (ICG) for retinal angiography was necessary to illustrate the choroidal vasculature given ICG's longer excitation/emission wavelength profile and its greater luminal retention [10]. In the future, it may be interesting to use ICG for scleral angiography as this tracer may better image the deep scleral bed. Alternatively combining ICG with fluorescein is another option and has been shown to be valuable in detection of corneal and scleral vascular pathology in previous studies $[9,15]$.

Moreover, scleral angiography can also be attempted for multiple conditions. While initially described for generalized scleritis and now possibly for tumor evaluation, one could consider evaluating scleral angiography as related to focal scleritis, severe intraocular uveitis with spillover into the sclera, pterygium growth and success of surgical removal, vascular communications for trabeculectomies, or for congenital disorders of the sclera.

For ocular oncology, we plan to apply scleral angiography to a wider number of patients. First, we will investigate a broader subset of malignant and benign tumors to better establish the range of pathology that can be seen with scleral angiography. In parallel, scleral angiography will also be applied to patients referred for sentinel vessel evaluation with or without the presence of a tumor. The question of when to use tumor detection technologies in the absence of obvious disease is a challenge for all diagnostic modalities. A key hint is often the potential sentinel vessel. The presence of a single large and tortuous vessel diving into the sclera over the ciliary body is a long-established clue that a tumor, which requires more vascular support than the ciliary body, may be present [4]. The presence of a suspicious looking vessel is often the sole impetus for initiating an ocular oncology workup that is likely to involve OCT and/or UBM. By evaluating patients with sentinel vessels, we can apply scleral angiography in cases without overt vascular changes seen in patient 1 and possibly identify a patient with a ciliary body tumor with greater normal appearing sclera yet abnormal scleral angiography.

Furthermore, scleral angiography could be used for more than assisting in diagnosis. Slow growing and possibly benign tumors are less likely to give rise to vascular invasion. As such, normal scleral angiography in the setting of a tumor may also influence a decision to biopsy a tumor as opposed to enucleation. Ultimately, we hope that this described technique, while requiring further evaluation, may be a useful adjunct to the comprehensive ophthalmologist and ocular oncologist.

\section{Acknowledgements}

Grant support: National Institutes of Health, Bethesda, Md. [grant numbers K08EY024674 (A.S.H.); P30EY03040 (Doheny Vision Institute Imaging Core)], American 
Marvasti et al.: Anterior Segment Scleral Fluorescein Angiography in the Evaluation of Ciliary Body Neoplasm: Two Case Reports

Glaucoma Society Mentoring for Physician Scientists Award 2013 and 2014 (A.S.H.), American Glaucoma Society Young Clinician Scientist Award 2015.

\section{Statement of Ethics}

Written informed consents were obtained from the patients.

\section{References}

1 Hayton S, Lafreniere R, Jerry LM, Temple WJ, Ashley P: Ocular melanoma in Alberta: a 38 year review pointing to the importance of tumor size and tumor histology as predictors of survival. J Surg Oncol 1989;42:215-218.

2 Gamel JW, McCurdy JB, McLean IW: A comparison of prognostic covariates for uveal melanoma. Invest Ophthalmol Vis Sci 1992;33:1919-1922.

3 Marigo FA, Finger PT, McCormick SA, et al: Iris and ciliary body melanomas: ultrasound biomicroscopy with histopathologic correlation. Arch Ophthalmol 2000;118:1515-1521.

4 Garmizo G: Sentinel episcleral vessels. A clue to the diagnosis of asymptomatic ciliary body melanoma. J Am Optom Assoc 1984;55:599-600.

5 Bakri SJ, Singh AD, Lowder CY, et al: Imaging of iris lesions with high-speed optical coherence tomography. Ophthalmic Surg Lasers Imaging 2007;38:27-34.

-6 Jensen VA, Lundbaek K: Fluorescence angiography of the iris in recent and long-term diabetes. Preliminary communication. Acta Ophthalmol (Copenh) 1968;46:584-585.

7 Watson PG, Bovey E: Anterior segment fluorescein angiography in the diagnosis of scleral inflammation. Ophthalmology 1985;92:1-11.

-8 Watson P, Booth-Mason S: Fluorescein angiography in the differential diagnosis of sclerokeratitis. $\mathrm{Br}$ J Ophthalmol 1987;71:145-151.

-9 Nieuwenhuizen J, Watson PG, Emmanouilidis-van der Spek K, Keunen JE, Jager MJ: The value of combining anterior segment fluorescein angiography with indocyanine green angiography in scleral inflammation. Ophthalmology 2003;110:1653-1666.

-10 Keane PA, Sadda SR: Imaging chorioretinal vascular disease. Eye (Lond) 2010;24:422-427.

11 Peyman GA, Gremillion CM: Eye wall resection in the management of uveal neoplasms. Jpn J Ophthalmol 1989;33:458-471.

$\$ 12$ Finger PT: Plaque radiation therapy for malignant melanoma of the iris and ciliary body. Am J Ophthalmol 2001;132:328-335.

13 Maruyama Y, Kishi S, Kamei Y, Shimizu R, Kimura Y: Infrared angiography of the anterior ocular segment. Surv Ophthalmol 1995;39(suppl 1):S40-S48.

14 Ishibashi S, Tawara A, Sohma R, Kubota T, Toh N: Angiographic changes in iris and iridocorneal angle neovascularization after intravitreal bevacizumab injection. Arch Ophthalmol 2010;128:1539-1545.

-15 Anijeet DR, Zheng Y, Tey A, Hodson M, Sueke H, Kaye SB: Imaging and evaluation of corneal vascularization using fluorescein and indocyanine green angiography. Invest Ophthalmol Vis Sci 2012;53:650-658. 
Case Reports in

Ophthalmology
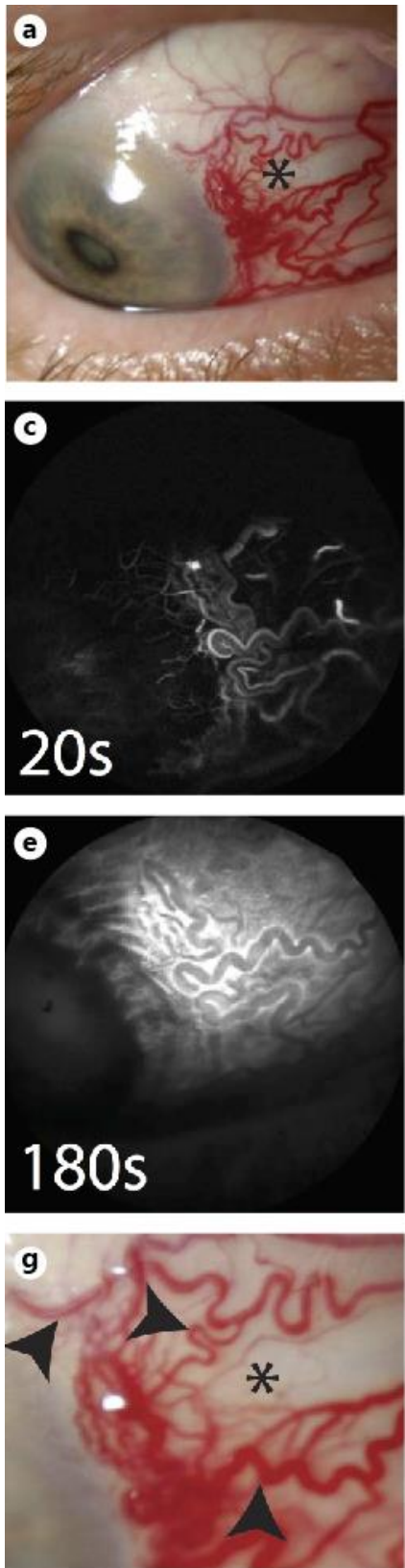

\begin{tabular}{l|l}
\hline Case Rep Ophthalmol 2016;7:30-38 \\
\hline DOI: 10.1159/000443603 & $\begin{array}{l}\text { c } 2016 \text { The Author(s). Published by S. Karger AG, Basel } \\
\text { www.karger.com/cop }\end{array}$
\end{tabular}

Marvasti et al.: Anterior Segment Scleral Fluorescein Angiography in the Evaluation of Ciliary Body Neoplasm: Two Case Reports
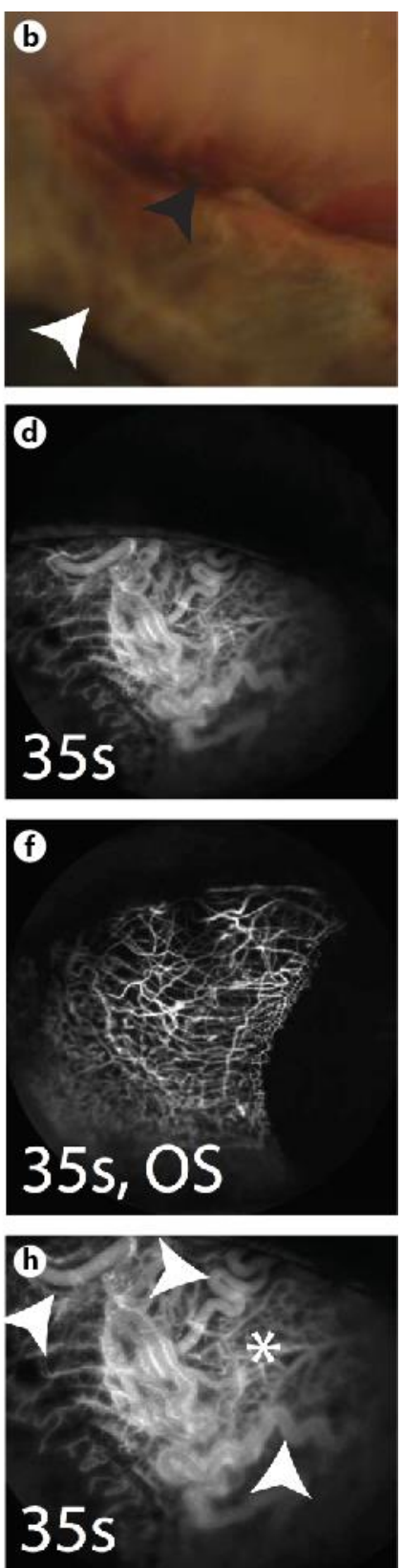

Fig. 1. Patient 1 (61-year-old Caucasian male). a Slit lamp photograph demonstrates abnormal nasal surface vasculature (asterisk). b Higher magnification slit lamp image shows the tumor (black arrowhead) with iris neovascularization (white arrowhead). c Scleral angiography early arterial phase shows laminar flow at $20 \mathrm{~s}$ [7]. d Arteriovenous phase shows various areas of pinpoint and linear hyperfluorescence at $35 \mathrm{~s}$ [7]. e Diffuse fluorescein signal is seen with leakage at $180 \mathrm{~s}$. $\mathrm{f}$ Scleral angiography at $35 \mathrm{~s}$ in the left eye demonstrates normal and organized vascular patterns with clear intervening regions. $\mathbf{g}$ Upon closer examination of the abnormal nasal region, vascular changes (black arrowheads) are seen intermixed with relatively normal appearing sclera (asterisk). $\mathbf{h}$ Scleral angiography focusing on the background of normal appearing sclera (white asterisk; black asterisk depicted in $\mathbf{g}$ ) shows a deep lacy complex of thick and abnormal vessels. White arrowheads in $\mathbf{h}$ correspond with black arrowheads in $\mathbf{g}$. OS = Left eye. 
Case Reports in

Ophthalmology

\begin{tabular}{l|l}
\hline Case Rep Ophthalmol 2016;7:30-38 \\
\hline DOI: 10.1159/000443603 & $\begin{array}{l}\text { @ 2016 The Author(s). Published by S. Karger AG, Basel } \\
\text { www.karger.com/cop }\end{array}$
\end{tabular}

Marvasti et al.: Anterior Segment Scleral Fluorescein Angiography in the Evaluation of Ciliary Body Neoplasm: Two Case Reports
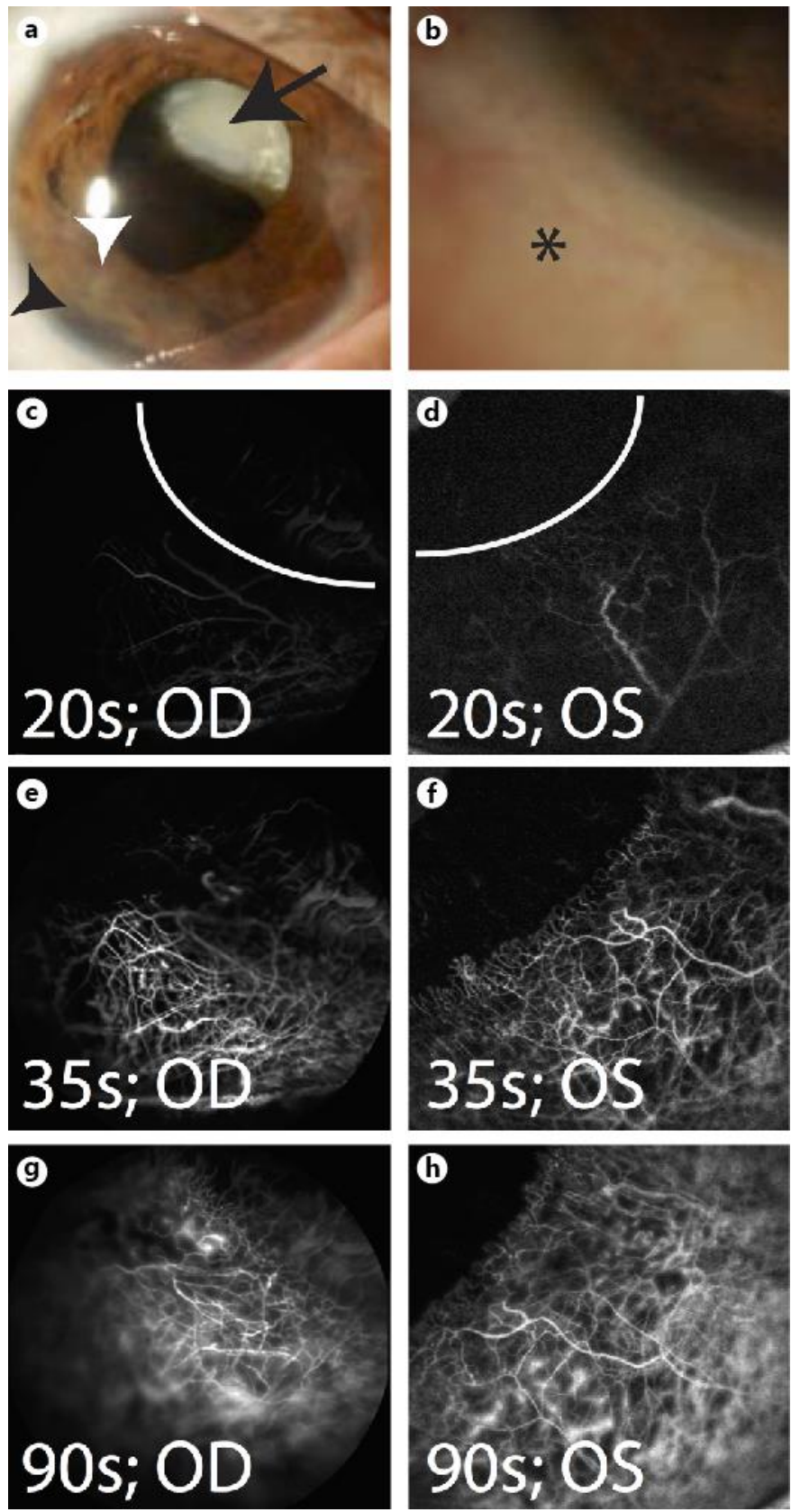

Fig. 2. Patient 2 (57-year-old Hispanic female). a Dilation reveals a superonasally displaced white cataractous lens (arrow), with tumor (white arrowhead) and iris root changes (black arrowhead). b Trace hyperemia of conjunctiva (asterisk). c, d Scleral angiography early arterial phase at $20 \mathrm{~s}$ [7]. e, $\mathbf{f}$ Arteriovenous phase with few pinpoint leakage at $35 \mathrm{~s}$ [7]. g, h Continued scleral angiographic signal with expanding areas of vascular leaks at $90 \mathrm{~s}$. Curved white lines denote the limbus. OD = Right eye; OS = left eye. 
Case Reports in

Ophthalmology
Case Rep Ophthalmol 2016;7:30-38

Marvasti et al.: Anterior Segment Scleral Fluorescein Angiography in the Evaluation of Ciliary Body Neoplasm: Two Case Reports
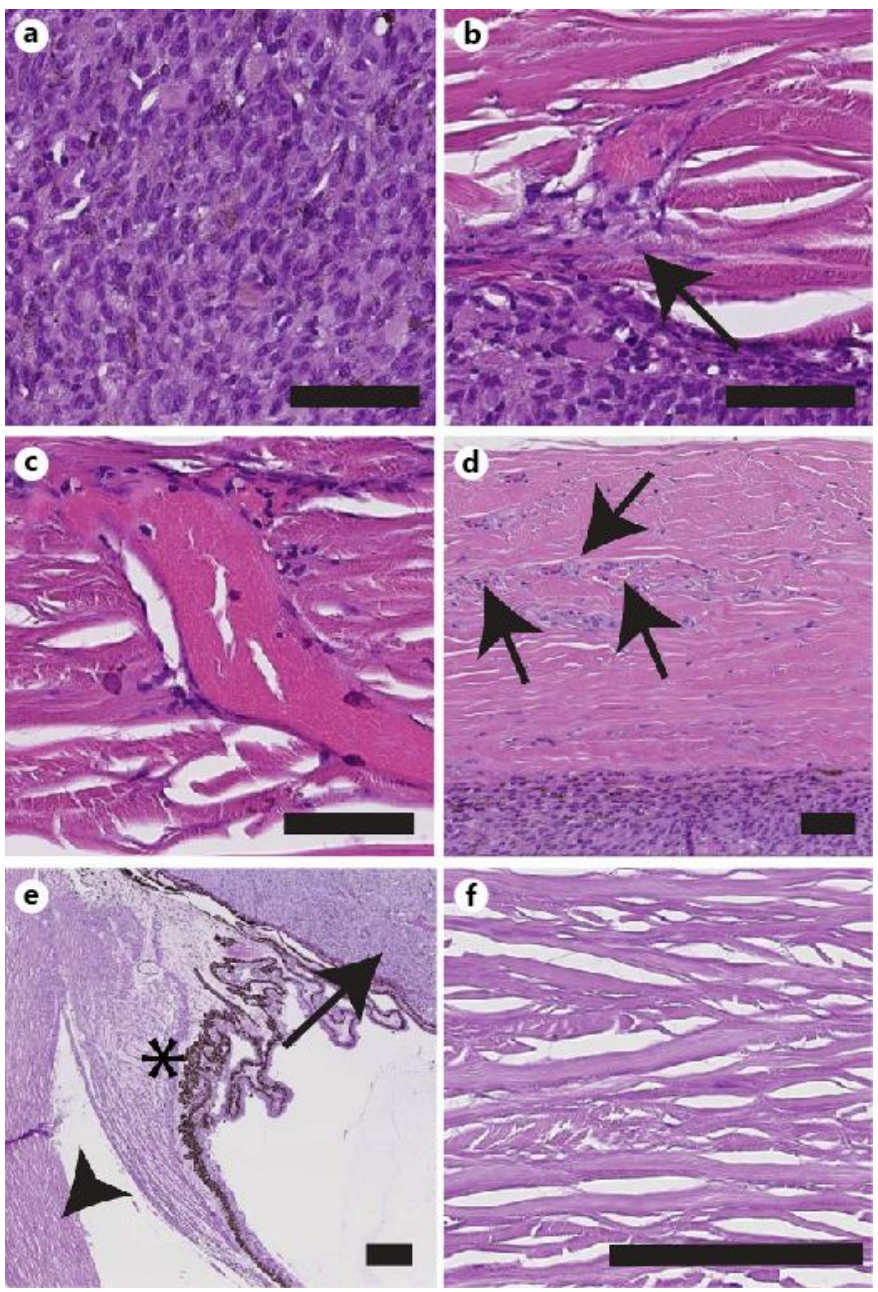

Fig. 3. Histological evaluation of tumors in patients $1(\mathbf{a}-\mathbf{d})$ and $2(\mathbf{e}, \mathbf{f})$. a Hematoxylin-eosin (HE) of patient 1's tumor demonstrates spindle cells and pigment deposition from the ciliary body melanoma. b Tumor invasion at the tumor/scleral border into the inner scleral bed (arrow). c An abnormal and large scleral vessel with associated tumor cells. $\mathbf{d}$ More subtle vessels (arrows) with associated abnormal cells in otherwise normal appearing sclera. Scale bars in $\mathbf{a}-\mathbf{d}=30 \mu \mathrm{m}$. e HE of patient 2's tumor demonstrates that its location (arrow) is separated from the scleral wall (arrowhead) by the ciliary body (asterisk). $\mathbf{f}$ Normal appearing sclera in patient 2's right eye. Scale bars in $\mathbf{e , ~} \mathbf{f}=100 \mu \mathrm{m}$. 